\title{
Sunspot seismic halos generated by fast MHD wave refraction
}

\author{
E. Khomenko ${ }^{1,2}$ and M. Collados ${ }^{1}$ \\ 1 Instituto de Astrofísica de Canarias, C/ vía Láctea, s/n, 38205 Tenerife, Spain \\ e-mail: khomenko@iac.es \\ 2 Main Astronomical Observatory, NAS, 03680 Kyiv, Ukraine
}

Received 30 July 2009 / Accepted 8 September 2009

ABSTRACT

\begin{abstract}
Aims. We suggest an explanation for the high-frequency power excess surrounding active regions known as seismic halos. Methods. We use numerical simulations of magneto-acoustic wave propagation in a magnetostatic sunspot model.

Results. We propose that seismic halos can be caused by the additional energy injected by high-frequency fast mode waves refracted in the higher atmosphere due to the rapid increase of the Alfvén speed. Our model qualitatively explains the magnitude of the halo and allows us to make predictions of its behavior that can be checked in future observations.
\end{abstract}

Key words. magnetohydrodynamics (MHD) - Sun: magnetic fields - Sun: oscillations - Sun: helioseismology

\section{Introduction}

Almost since the discovery of the 5-min solar oscillations, it has been well known that the oscillation power is reduced by some 40-60\% in the photospheres of sunspots (Lites et al. 1982; Abdelatif et al. 1986; Brown et al. 1992; Title et al. 1992; Hindman \& Brown 1998). Later it was found that the highfrequency non-trapped wave power shows a suspicious enhancement in rings surrounding active regions, both in the photosphere (Brown et al. 1992) and in the chromosphere (Braun et al. 1992; Toner \& Labonte 1993). These power enhancements are known as "halos". Their observational properties can be summarized as follows:

(i) the power enhancement is observed at high frequencies, between 5.5 and $7.5 \mathrm{mHz}$ for waves that are usually nontrapped in the non-magnetic quiet Sun;

(ii) the acoustic power measured in halos is higher than in the nearby quite Sun by about 40-60\% (Hindman \& Brown 1998; Braun \& Lindsey 1999; Donea et al. 2000; Jain \& Haber 2002; Nagashima et al. 2007);

(iii) the halos are observed at intermediate longitudinal magnetic fluxes $\langle B\rangle=50-300 \mathrm{G}$, while the acoustic power is usually reduced at all frequencies at larger fluxes (Hindman \& Brown 1998; Thomas \& Stanchfield 2000; Jain \& Haber 2002);

(iv) the radius of the halo increases with height. In the photosphere the halos are located at the edges of active regions, while in the chromosphere they extend to a large portion of the nearby quiet Sun (Brown et al. 1992; Braun et al. 1992; Thomas \& Stanchfield 2000);

(v) the power increase in the halo is qualitatively similar in sunspots, pores and plages;

(vi) significant reflection of the upcoming acoustic radiation at 5-6 $\mathrm{mHz}$ is detected in active regions, unlike the behavior of such high-frequency waves in the quiet Sun (Braun \& Lindsey 2000).
While several plausible mechanisms have been proposed to explain the acoustic power reduction for the strongest fields in active regions (e.g. MHD mode conversion; Cally \& Bogdan 1997), no accepted theory exists to explain the power enhancement in acoustic halos. The increase of the high-frequency acoustic emission, initially proposed by Brown et al. (1992) and Braun et al. (1992), seems not to be confirmed observationally, since the observed continuum intensity does not show the halo effect (Hindman \& Brown 1998; Jain \& Haber 2002). Alternatively, the latter authors propose that the velocity in the surroundings of active regions may become field-aligned and some type of incompressible wave may be responsible for halos. However, this lacks any observational evidence. Recently, Kuridze et al. (2008) suggested yet another mechanism based on acoustic waves trapped in field-free atmospheres lying below small-scale magnetic canopies of network cores and active regions. Interestingly, halos were observed recently in MHD simulations of waves in magnetic structures by Hanasoge (2008) and Shelyag et al. (2009). Based on his simulations, Hanasoge (2009) suggests that the power enhancement in halos is due to magnetic field induced mode mixing resulting in preferential scattering from low to high wave numbers.

In this Letter, we propose a mechanism based on the fast MHD mode refraction in the vicinity of the transformation layer (where the Alfvén speed $v_{\mathrm{A}}$ is equal to the sound speed $c_{\mathrm{S}}$ ) that is able to explain several observational properties of halos. In addition, we predict some new properties that can be obtained from observations in the future to confirm or refute this explanation.

\section{Description of methods}

We perform 2D numerical experiments that are similar to those of Khomenko et al. (2009) to study the adiabatic propagation of magneto-acoustic waves excited by a single source located in sub-photospheric layers of a magneto-static sunspot model. The numerical MHD code is described in detail in Khomenko \& Collados (2006); Khomenko et al. (2008). The unperturbed 

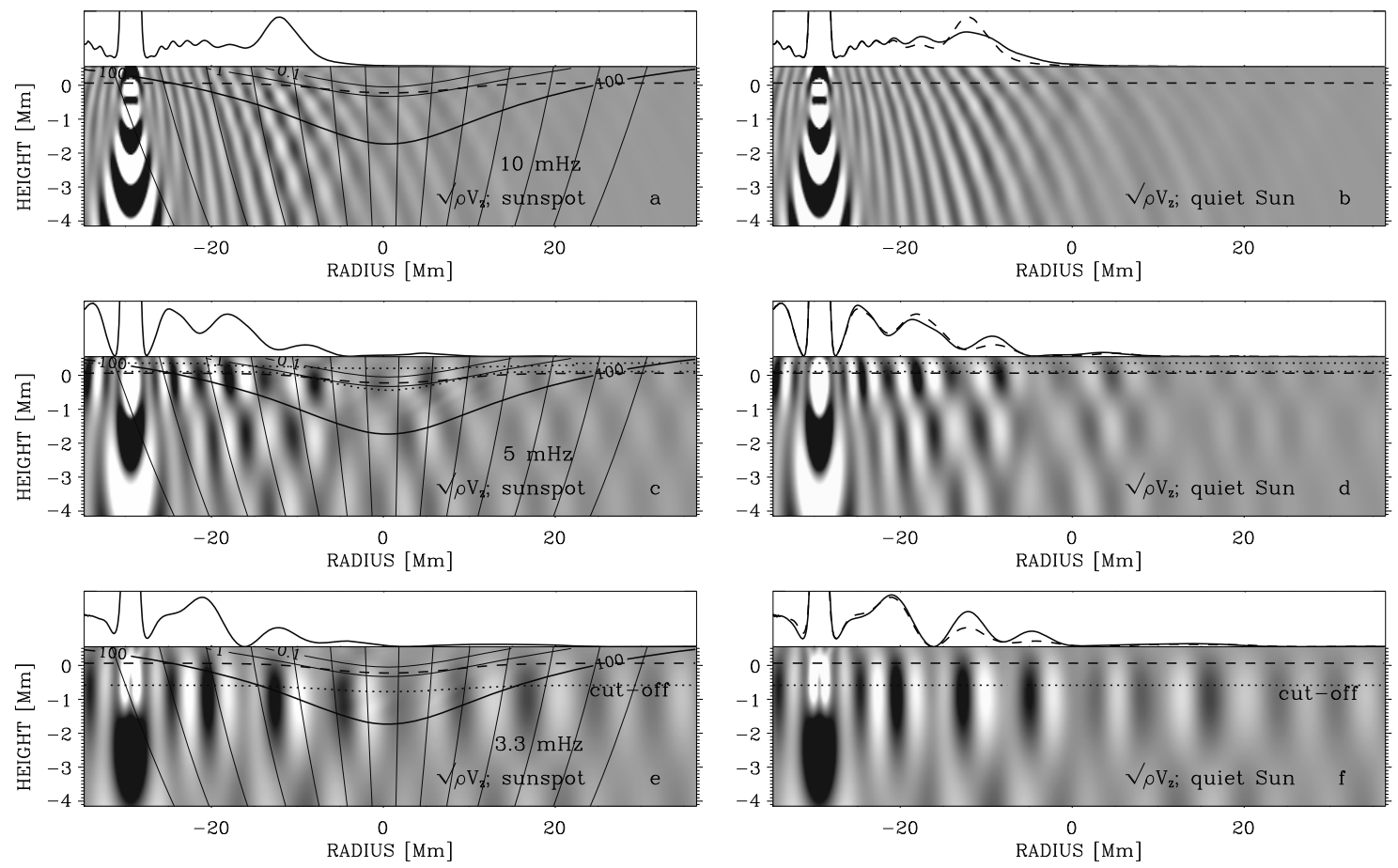

Fig. 1. Snapshots of the vertical velocity (scaled with the factor $\sqrt{\rho}$ ) at an elapsed time of $60 \mathrm{~min}$ in the simulations with a harmonic source placed in the sunspot model (left panels) and in the quiet Sun model (right panels). Panels from top to bottom: source periods 100,200 and 300 s. The upper plots on each panel give the rms amplitude distribution at the line formation level. The curve from the sunspot simulations is repeated with a dashed line in the right panels. The contours of constant $c_{\mathrm{S}}^{2} / v_{\mathrm{A}}^{2}$ are marked with numbers. The dashed-line contours indicate the "line formation" heights in both models. Dotted lines: height where the period of the source coincides with the cut-off period. The black inclined lines are magnetic field lines.

magnetostatic sunspot model is taken from Khomenko \& Collados (2008). The simulation domain has $80 \times 10.5 \mathrm{Mm}^{2}$ in horizontal and vertical directions, respectively, with a resolution of $\mathrm{d} x=0.15 \mathrm{Mm}$ and $\mathrm{d} z=0.05 \mathrm{Mm}$. The whole domain contains a magnetic field, but it becomes weak and dynamically unimportant further than $\sim 20 \mathrm{Mm}$ from the sunspot axis. The maximum field strength in the photosphere is around $1 \mathrm{kG}$. Our sunspot model has a Wilson depression. Using the SIR radiative transfer code (Ruiz Cobo \& del Toro Iniesta 1992), we calculated the optical depth scale, $\log \tau_{5}$, from the known distribution of thermodynamic parameters in geometrical height for each horizontal point of the MHS sunspot model. The photospheric level defined by the optical depth scale $\log \tau_{5}=0$ is located $300 \mathrm{~km}$ deeper at the sunspot axis compared to its location $40 \mathrm{Mm}$ away from the axis (see Figs. 1 and 2 in Khomenko et al. 2009). We define two reference levels of optical depth: $\log \tau_{5}=0$ ("photosphere") and $\log \tau_{5}=-1.6$ ("line formation"). At a horizontal distance $X=40 \mathrm{Mm}$ from the sunspot axis, the "photosphere" is located $500 \mathrm{~km}$ below the top boundary of our simulation domain. The "line formation" level is located $160 \mathrm{~km}$ above the "photosphere". At $20 \mathrm{Mm}$ from the axis, the $v_{\mathrm{A}}=c_{\mathrm{S}}$ level is above the "line formation" level by about $300 \mathrm{~km}$, and at the axis it is located some $200 \mathrm{~km}$ below.

In the first set of experiments the source is placed at three different horizontal distances $X_{0}=20,30$ and $35 \mathrm{Mm}$ from the sunspot axis and at $Z_{0}=-700 \mathrm{~km}$ below the photosphere. The temporal behavior of the source is described by a Ricker wavelet (Parchevsky \& Kosovichev 2009; Khomenko et al. 2009) with a central frequency of $3.3 \mathrm{mHz}$. In the second set of experiments the source is placed at $X_{0}=30 \mathrm{Mm}$ and $Z_{0}=-450 \mathrm{~km}$, and it is harmonic and continuous in time with three different frequencies $v=10,5$ and $3.3 \mathrm{mHz}$. In all the cases the sunspot simulations are accompanied by non-magnetic simulations in the modified model S of Christensen-Dalsgaard et al. (1996, see Parchevsky \& Kosovichev 2007) with the same properties of the source and numerical treatment. The duration of simulations is about two physical hours.

\section{Results}

Figure 1 shows snapshots of the vertical velocity in simulations with the harmonic source. When the wave frequency is well above the cut-off frequency $(v=10 \mathrm{mHz})$, the waves are propagating in the quiet Sun (Fig. 1b). This situation is different in the sunspot model. Significant reflections can be seen in Fig. 1a as an interference wave pattern around $X=-20$ : $-10 \mathrm{Mm}$. The reflection of the high-frequency waves is produced in the vicinity of the $v_{\mathrm{A}}=c_{\mathrm{S}}$ transformation layer. The fast (acoustic-like) waves generated by the source are transmitted as fast (magnetic) waves in the upper atmosphere where the Alfvén speed is higher that the sound speed $\left(v_{\mathrm{A}}>c_{\mathrm{S}}\right)$. In the magnetically dominated layers these waves are refracted (see Khomenko \& Collados 2006) and are returned to the sub-photospheric layers where they interfere with the waves coming from the source. This behavior of waves is clearly seen in the movie of this simulation, attached as on-line material to this paper. The rms vertical velocity amplitude measured at the line formation level (dashed curves in Figs. 1a and b) shows a suspicious bump to the left of the location where the $\log \tau_{5}=-1.6$ contour crosses the $v_{\mathrm{A}}=c_{\mathrm{S}}$ contour (see upper plots in each panel). This bump is absent in the quiet Sun simulation. We propose that the increase in the high-frequency power in halos surrounding active regions can be produced by the additional energy injected by the fast mode waves refracted in the magnetically dominated layers 

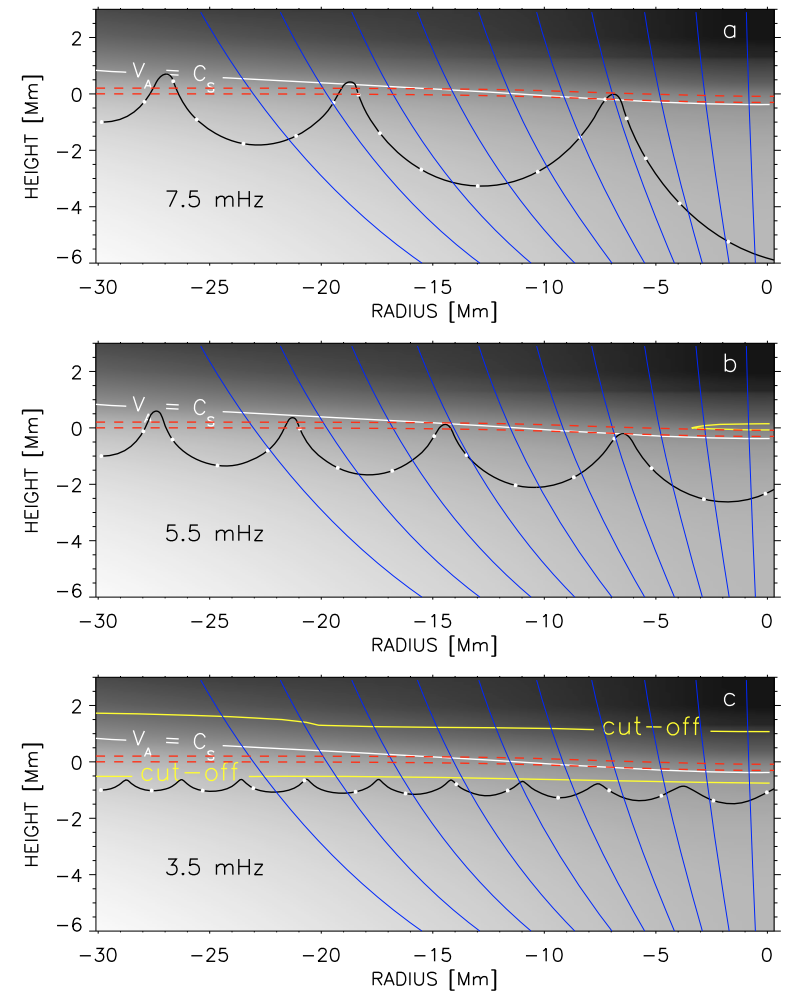

Fig. 2. Wave paths of the fast mode launched from the lower turning point at $X=-30 \mathrm{Mm}, Z=-1 \mathrm{Mm}$ propagating through the sunspot model for frequencies $7.5,5.5$ and $3.5 \mathrm{mHz}$ (from top to bottom). For clearness only the upper part of the model is shown (not to scale). Blue inclined lines are magnetic field lines. The yellow contours mark the layer where the wave frequency is equal to the cut-off frequency. The white contours are $v_{\mathrm{A}}=c_{\mathrm{S}}$. The background image is $\log _{10}\left(v_{\mathrm{A}}\right)$. Each white dot on the trajectory is separated by $3 \mathrm{~min}$ in time. The red dashed lines are the "photosphere" and the "line formation" levels.

back to sub-photospheric layers. The presence of the upward and downward propagating wave energy manifests itself as the wave interference pattern. Note that this mechanism does not necessarily imply that the high-frequency fast waves are trapped in sunspots, since a part of their energy can leak into the slow mode waves after each mode transformation near the $v_{\mathrm{A}}=c_{\mathrm{S}}$ layer. However, this mechanism produces significant reflections of the high-frequency waves that otherwise propagate in the quiet Sun.

When the wave frequency is below the photospheric cut-off frequency ( $v=3.3 \mathrm{mHz}$, Figs. 1e, f) the fast mode waves become evanescent before reaching the transformation $v_{\mathrm{A}}=c_{\mathrm{S}}$ layer. In this case neither refraction nor interference can be produced because of the absence of fast to fast mode transmission. Figures 1e, f (upper plots) shows that the rms. amplitude distributions are very similar in the sunspot and the quiet Sun simulations and that the amplitudes in the sunspot case are always lower than in the quiet Sun.

The $v=5 \mathrm{mHz}$ case (Figs. 1c,d) shows an intermediate situation, where the waves are reflected both due to cut-off effects and magnetic effects. In this case, the cut-off height (dotted lines) almost coincides with the $v_{\mathrm{A}}=c_{\mathrm{S}}$ height. Some power excess is still present in the sunspot case compared to the quiet Sun. The bump is weaker and is now located nearer the source as the interference happens at another location due to the different wavelength. Note that the rms velocity distribution is less smooth for $v=5$ and $3.3 \mathrm{mHz}$ compared to $v=10 \mathrm{mHz}$, as several nodes are present in the horizontal (and vertical) direction due to the evanescent character of waves.

The eikonal solution for the fast mode wave (Cally 2006; Moradi \& Cally 2008; Khomenko et al. 2009) allows us to develop a more complete physical picture of the wave behavior at different frequencies. Figure 2 gives the wave paths of the fast mode waves launched from their lower turning point (that roughly coincides with the location of the source in the simulations). At high frequencies $(5.5-7.5 \mathrm{mHz})$, the waves penetrate higher in the atmosphere above the line formation layer where they are refracted back down due to the presence of the magnetic field. In the low-frequency case $(3.5 \mathrm{mHz}$, Fig. 2c) the waves are sharply reflected from the cut-off layer and are less affected by the magnetic field. Note that the power excess (halos) at high frequency should form at distances where the refraction of the fast mode occurs above the line formation layer, i.e. where the $v_{\mathrm{A}}=c_{\mathrm{S}}$ layer lies above the photosphere. Otherwise it would not be detected in spectral observations. This happens in regions of intermediate field strengths. Under no circumstances can the halo form in the "umbral" zone with strong fields where $v_{\mathrm{A}}$ is higher than $c_{\mathrm{S}}$ in the photosphere.

A more realistic situation with the source emitting a spectrum of waves is considered in Fig. 3. In this figure we compare the wavelet source simulations with different source locations. We represent the ratio between the photospheric Fourier power in the sunspot and in the quiet Sun at different frequencies as a function of horizontal distance. We conclude that, independently of the position of the source, the overall picture is very similar especially for the sources at -35 and $-30 \mathrm{Mm}$. For the source located at $-20 \mathrm{Mm}$ from the axis, the magnetic field already plays a role and modifies the properties of the source compared to the purely non-magnetic case, thus contaminating the detailed picture. Still, even in this case, a halo power increase is present around $-10 \mathrm{Mm}$, being weaker than in the other two cases. Figure 3 shows a power excess at high frequencies above $5.5 \mathrm{mHz}$ at distances $-20:-10 \mathrm{Mm}$ from the sunspot axis (the latter position coincides with the location where $v_{\mathrm{A}}=c_{\mathrm{S}}$ in the photosphere). At low frequencies, a power deficit is observed in the sunspot. The power deficit is also present at high frequencies in the "umbral" region. The power averaged over both lowfrequency and high-frequency bands shows a 40-60\% decrease in the umbra relative to the quiet Sun. This decrease is because part of the source energy is lost after the multiple mode transformations (see Cally \& Bogdan 1997). In the high-frequency band, however, there is an excess of power up to some $40-50 \%$. Note that the magnitude of the halo in our simulations is in good agreement with the observed one (e.g. Hindman \& Brown 1998).

\section{Discussion and conclusions}

Our results indicate that the halo effect happens in a natural way due to additional energy input from the high-frequency fast mode waves produced after their refraction. The halo is produced in the photospheric regions where the field is intermediate, implying that the Alfvén speed is lower than the sound speed. The halo is not observed at low frequencies because these waves are already reflected below the transformation layer. The halo is not observed in the umbral part of the sunspot because the refraction happens below the layer visible in spectral line observations.

In our simulations, the halo is observed at the periphery of the strong field zone where the field is inclined by some 30-40 degrees. For sunspots with greater field strengths than considered here, the level $c_{\mathrm{S}}=v_{\mathrm{A}}$ would be located deeper and would intersect the $\log \tau_{5}=0$ level further from the sunspot axis. 

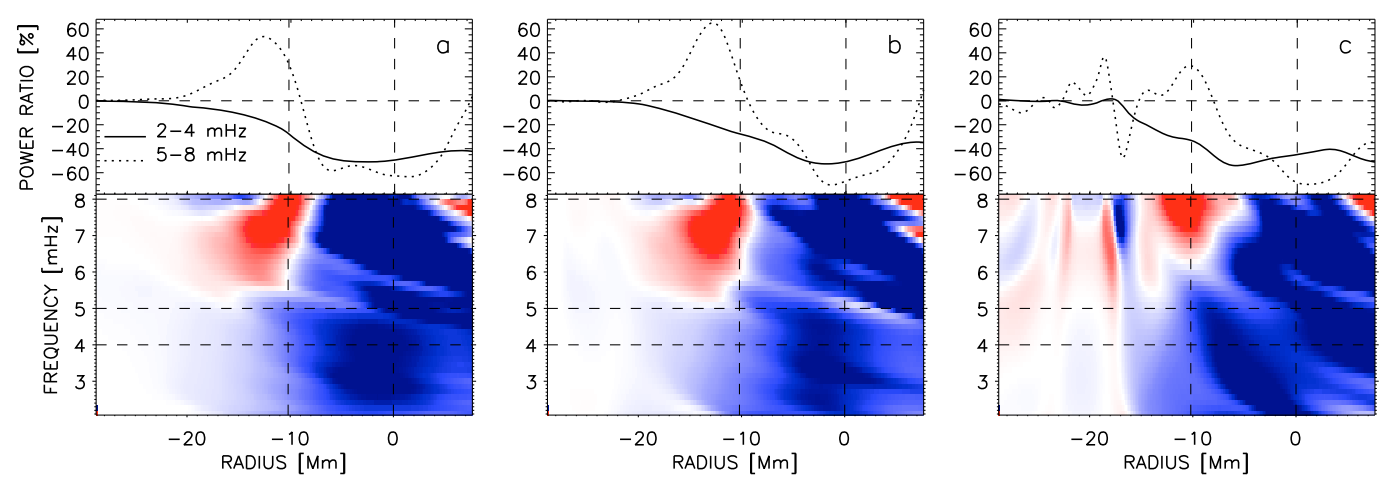

Fig. 3. Ratio between the photospheric wave power in the sunspot model relative to the quiet Sun model $\left(\left(P_{\text {spot }}-P_{\text {quiet }}\right) / P_{\text {quiet }}\right)$ as a function of horizontal position and frequency for three simulations with the wavelet source located at $\left.\left.X_{0}=-35 \mathbf{a}\right),-30 \mathbf{b}\right)$ and -20 c) $\mathrm{Mm}$. The color scale at the bottom panels ranges from -50 to $50 \%$ (red means power excess). The upper panels show the power ratio averaged over the low-frequency and high-frequency bands (marked on the figure). The vertical dashed line located at $X=0$ marks the sunspot axis. Another dashed line marks the location where $v_{\mathrm{A}}=c_{\mathrm{s}}$ in the photosphere.

Thus, for models with more intense magnetic fields, we expect that the halo will appear at greater distances from the sunspot axis. Also, by increasing the magnetic field inclination in the penumbra of the sunspot model, the fast to fast mode transmission will be more efficient (Cally 2006) and we can expect the magnitude of the halo to be somewhat larger.

Based on our model, we can speculate about some observed properties of the halo as well as others (still undetected) that may be interesting to observe in the future.

(i) Several observations indicate that the radius of the halo increases with height (Brown et al. 1992; Braun et al. 1992; Thomas \& Stanchfield 2000). This effect can be qualitatively explained by our model. As follows from e.g. Fig. 1, with increasing height, the $v_{\mathrm{A}}=c_{\mathrm{S}}$ layer is located at progressively greater distances from the sunspot axis. Observed in chromospheric lines, the condition necessary to detect the halo (i.e. that the line formation layer lies below the $v_{\mathrm{A}}=c_{\mathrm{S}}$ layer) would be fulfilled at greater distances from the sunspot axis, and, in a natural way, this would produce halos with larger radius. We can predict that, after some height in the chromosphere where the whole atmosphere is magnetically dominated, the halos should disappear.

(ii) Our model also explains qualitatively the observations of Braun \& Lindsey (2000) who detected significant reflection of the high-frequency waves in active regions.

(iii) The horizontal velocity component (not shown in this paper) shows a stronger magnitude of the halo effect as the waves propagate nearly horizontally at the heights where they are refracted. Thus, we suggest that the magnitude of the halo in off-center observations should be stronger. We are aware of only one observation of this kind (Toner \& Labonte 1993), where apparently no change of the halo magnitude was detected. However, more observations are required to confirm/discard this conclusion.

(iv) Since the magnitude of the mode transformation and reflection at the $v_{\mathrm{A}}=c_{\mathrm{S}}$ layer depends on the magnetic field inclination (Cally 2006), we can speculate that halos, when detected with high resolution observations, should show fine structure effects in active region penumbral filaments, this effect being more pronounced for horizontal fields.

Acknowledgements. This research has been funded by the Spanish Ministerio de Educación y Ciencia through projects AYA2007-63881 and AYA2007-66502.

\section{References}

Abdelatif, T. E., Lites, B. W., \& Thomas, J. H. 1986, ApJ, 311, 1015 Braun, D. C., \& Lindsey, C. 1999, ApJ, 513, L79

Braun, D. C., \& Lindsey, C. 2000, Sol. Phys., 192, 307

Braun, D. C., Lindsey, C., Fan, Y., \& Jefferies, S. M. 1992, ApJ, 392, 739

Brown, T. M., Bogdan, T. J., Lites, B. W., \& Thomas, J. H. 1992, ApJ, 394, L65 Cally, P. 2006, Phil. Trans. R. Soc. A, 364, 333

Cally, P. S., \& Bogdan, T. J. 1997, ApJ, 486, L67

Christensen-Dalsgaard, J., Dappen, W., Ajukov, S. V., et al. 1996, Science, 272, 1286

Donea, A.-C., Lindsey, C., \& Braun, D. C. 2000, Sol. Phys., 192, 321

Hanasoge, S. M. 2008, ApJ, 680, 1457

Hanasoge, S. M. 2009, A\&A, in press

Hindman, B. W., \& Brown, T. M. 1998, ApJ, 504, 1029

Jain, R., \& Haber, D. 2002, A\&A, 387, 1092

Khomenko, E., \& Collados, M. 2006, ApJ, 653, 739

Khomenko, E., \& Collados, M. 2008, ApJ, 689, 1379

Khomenko, E., Collados, M., \& Feliipe, T. 2008, Sol. Phys., 251, 589

Khomenko, E., Kosovichev, A., Collados, M., Parchevsky, K., \& Olshevsky, V. 2009, ApJ, 694, 411

Kuridze, D., Zaqarashvili, T. V., Shergelashvili, B. M., \& Poedts, S. 2008, Annales Geophysicae, 26, 2983

Lites, B. W., White, O. R., \& Packman, D. 1982, ApJ, 253, 386

Moradi, H., \& Cally, P. 2008, Sol. Phys., 215, 309

Nagashima, K., Sekii, T., Kosovichev, A. G., et al. 2007, PASP, 59, S631

Parchevsky, K. V., \& Kosovichev, A. G. 2007, ApJ, 666, 547

Parchevsky, K. V., \& Kosovichev, A. G. 2009, ApJ, 694, 573

Ruiz Cobo, B., \& del Toro Iniesta, J. C. 1992, ApJ, 398, 375

Shelyag, S., Zharkov, S., Fedun, V., Erdélyi, R., \& Thompson, M. J. 2009, A\&A, 501,735

Thomas, J. H., \& Stanchfield, D. C. H. 2000, ApJ, 537, 1086

Title, A. M., Topka, K. P., Tarbell, T. D., et al. 1992, ApJ, 393, 782

Toner, C. G., \& Labonte, B. J. 1993, ApJ, 415, 847 